\title{
CRISPR-Cas13d Exhibits Robust Antiviral Activity Against Seneca Valley Virus
}

OPEN ACCESS

Edited by:

Shuofeng Yuan,

The University of Hong Kong, Hong Kong SAR, China

Reviewed by:

Haoran Sun,

University of Hong Kong, China

Bodan $\mathrm{Hu}$,

The University of Hong Kong,

Hong Kong SAR, China

${ }^{*}$ Correspondence:

Yan-Dong Tang

tangyandong2008@163.com; tangyandong@caas.cn Fandan Meng

mengfandan@caas.cn Xue-Hui Cai

caixuehui139@163.com

tThese authors have contributed equally to this work

Specialty section: This article was submitted to Virology,

a section of the journal Frontiers in Microbiology

Received: 14 December 2021 Accepted: 26 January 2022 Published: 14 February 2022

Citation:

Zhang $Y-Y$, Sun $M-X$, Lian $Y$, Wang $T-Y$, Jia $M-Y$, Leng $C$, Chen $M$, Bai $Y$-Z, Meng F, Cai X-H and Tang Y-D (2022) CRISPR-Cas $13 d$ Exhibits Robust Antiviral Activity Against Seneca Valley Virus.

Front. Microbiol. 13:835040. doi: 10.3389/fmicb.2022.835040

\section{Yu-Yuan Zhang ${ }^{1 t}$, Ming-Xia Sun ${ }^{1 t}$, Yuexiao Lian ${ }^{2 t}$, Tong-Yun Wang ${ }^{1}$, Mei-Yu Jia ${ }^{1}$, Chaoliang Leng ${ }^{3}$, Meng Chen ${ }^{1}$, Yuan-Zhe Bai ${ }^{1}$, Fandan Meng ${ }^{1 *}$, Xue-Hui Cai ${ }^{1 *}$ and Yan-Dong Tang ${ }^{1 *}$}

'State Key Laboratory of Veterinary Biotechnology, Harbin Veterinary Research Institute of Chinese Academy of Agricultural Sciences, Harbin, China, ${ }^{2}$ Guangdong Laboratory Animals Monitoring Institute and Guangdong Key Laboratory of Laboratory Animals, Guangzhou, China, ${ }^{3}$ Henan Provincial Engineering and Technology Center of Animal Disease Diagnosis and Integrated Control, Nanyang Normal University, Nanyang, China

In recent years, Seneca Valley virus (SWV) as a newly identified pathogen of porcine vesicular disease spread quickly and has posed a potential threat to the swine industry in several countries resulting in economic losses. Considering the evolution of SW, attention should be given to controlling SW epidemics. So far there are no commercial vaccines or drugs available to combat SV. Therefore, development of strategies for preventing and controlling SW infection should be taken into account. In the current study, we evaluated whether the CRISPR-Cas13d system could be used as a powerful tool against SW infection. Besides, selected crRNAs showed different capacity against SW infection. Our study suggests the CRISPR-Cas13d system significantly inhibited SW replication and exhibited potent anti-SW activity. This knowledge may provide a novel alternative strategy to control epidemics of SW in the future.

Keywords: CRISPR-Cas13d, CasRx, SVV, antiviral, virus

\section{INTRODUCTION}

Seneca Valley virus (SVV), also known as Senecavirus A, causes vesicular disease in pigs, and it is a non-enveloped, single-stranded RNA virus that belongs to the genus Senecavirus in the family Picornaviridae (Hales et al., 2008). SVV was first isolated and identified as a cell culture contaminant in the United States in 2002 (Reddy et al., 2007). Initially, SVV was mainly used as a potential oncolytic virus in cancer therapy, and there was no evidence that SVV was pathogenic to farm animals (Burke, 2016). However, in recent years, SVV has been reported to cause outbreaks in many countries and is associated with porcine vesicular disease (Hause et al., 2016; Leme et al., 2016; Zhao et al., 2017; Zhu et al., 2017; Zhang et al., 2018). The virulence of SVV seems to have increased when it evolved in pigs, and some newly emerged SVV strains lead to acute death of neonatal piglets (Leme et al., 2016, 2017). Currently, there are no commercial vaccines or drugs available to combat SVV.

The CRISPR-Cas system is recognized as the "adaptive" immune system of bacteria or archaea, protecting these organisms from invasions of viral infection, plasmid transfer, and foreign DNA interference (Horvath and Barrangou, 2010; Liao et al., 2021). CRISPR-Cas9 system has been reported to inhibit DNA virus replication effectively (Tang et al., 2016, 2017, 2018). Recently, the 
CRISPR-Cas13d system is an RNA-guided, RNA-targeting CRISPR system that exhibits high knockdown efficiency and strong specificity compared to RNA interference (Konermann et al., 2018). CRISPRassociated RNAs (crRNAs) contain an approximately 22-nt spacer sequence that guides the Cas13d protein to target RNA for degradation. Cas13d derived from Ruminococcus flavefaciens XPD3002, also known as CasRx, shows the best knockdown activity in mammalian cells (Konermann et al., 2018). Recently, the CRISPR-Cas13d system has been used to combat several RNA viruses, including SARS-CoV-2, and exhibits potent antiviral activity (Abbott et al., 2020; Nguyen et al., 2020; Lin et al., 2021).

\section{MATERIALS AND METHODS}

\section{Cells, Virus, Reagents, and Plasmids}

Swine testicle (ST) cells and human embryonic kidney (HEK293T) cells were maintained in Dulbecco's modified Eagle medium (DMEM; Thermo Fisher, United States) supplemented with 10\% fetal bovine serum as previously described (Yang et al., 2020, 2021).

The Senecavirus A strain (GD strain) was isolated and sequenced in our laboratory (unpublished data). The virus was propagated and titered in ST cells. Viral titers were determined by a $50 \%$ tissue culture infectious dose (TCID50) assay in ST cells. Viral titers were calculated using the Reed and Muench method (Reed and Muench, 1938).

The anti-HA monoclonal antibody (H9658) was purchased from Sigma. The VP2 monoclonal antibody was produced and preserved by our laboratory. The secondary antibodies used for immunofluorescence analyses were Alexa Fluor 568 goat anti-rabbit or mouse IgG (H+L; Thermo Fisher, United States). The cellular nuclei were stained with 4',6-diamidino-2phenylindole (DAPI, Beyotime, Beijing, China).

\section{CRISPR-Cas13d System}

The RfxCas13d expression vector pXR001 EF1a-CasRx-2A-EGFP was obtained from Addgene (Addgene, United States). To construct CasRx-del-NSL, two individual nuclear localization signals were removed from the EF1a-CasRx-2A-EGFP plasmid. Briefly, overlapping PCR was performed, and the construct was recloned into the original vector. The four specific crRNA targeting sites were as follows: crRNA\#1: TGCATTTCCATAAG AGAGAGCGC, crRNA\#2: CATTTCCATAAGAGAGAGCGCTC, crRNA\#3: CCAACATAGAAACAGATTGCAGC, and crRNA\#4: ATCGTCAGACATTTCCACCCACT. Guide RNA was synthesized as DNA oligos, and then, each crRNA was cloned into the pXR003 CasRx crRNA cloning backbone (Addgene, United States).

\section{Virus Replication Inhibition Assay}

HEK-293T cells were seeded in 12-well plates, and $1 \mu \mathrm{g}$ of CasRx and $1 \mu \mathrm{g}$ of the indicated crRNA expression vector were cotransfected into 293T cells. Twelve hours later, the cells were infected with SVV at an MOI of 0.01 . The virus was collected 24 and $48 \mathrm{hpi}$. The titers of SVV were determined by the TCID50 assay. IFA was performed as described in our previous work (Zhang et al., 2021).

\section{Colocalization Coefficients Analysis}

HEK-293T cells were seeded in 12-well plates, and $1 \mu \mathrm{g}$ of CasRx and $1 \mu \mathrm{g}$ of the indicated crRNA plasmid or mix pool (contain four indicated crRNA at equal ratio) were cotransfected into $293 \mathrm{~T}$ cells. Twelve hours later, the cells were infected with SVV at an MOI of 0.36 random selected images were analyzed by ImageJ. Manders' Colocalization Coefficients (Manders' $\mathrm{M}$ relate to EGFP) was analysis.

\section{Cell Viability Assay}

HEK293T cells were seeded in 96-well plates, and $0.05 \mu \mathrm{g}$ of the indicated crRNA plasmid (\#1, \#2, \#3, \#4, or Mix pool) and $0.05 \mu \mathrm{g}$ of CasRx were cotransfected into 293T cells. Viability of cells was determined using the Cell Counting Kit-8 (Dojindo, catalog number CK04). Ten microliter of the cck-8 solution was added after 24 or $48 \mathrm{~h}$ of incubation. Subject to incubation at $37^{\circ} \mathrm{C}$ for $2-4 \mathrm{~h}$, the absorbance at $450 \mathrm{~nm}$ was measured by An Absorbance Microplate Reader (The ELx808TM).

\section{Statistical Analysis}

Statistical significance was analyzed with one-way ANOVA and Tukey's multiple comparison test or the independent-samples Student's $t$-test in Origin GraphPad Prism 8.0 software. All differences with value of $p<0.05$ were considered statistically significant.

\section{RESULTS AND DISCUSSION}

In the current study, we tested whether the CRISPR-Cas13d system could be used as an effective antiviral strategy to combat SVV. The SVV strain in this study was isolated and sequenced in our laboratory (unpublished data). The genome structure of SVV is shown in Figure 1 (upper panel). To predict the possible crRNA, we first input the whole genome sequence into the predicted software, which is available at https://gitlab.com/ sanjanalab/cas13 (Wessels et al., 2020). In software screening, potential crRNA sequences were listed and ranked by their predicted standardized guide scores (Figure 1). We selected four crRNAs with high scores (two targeting P2C, one targeting $\mathrm{P} 3 \mathrm{~A}$, and one targeting RdRp) from the list for further investigation. Because SVV mainly undergoes replication and viral RNA synthesis in the cytoplasm rather than the nucleus (Flather and Semler, 2015), CasRx had to be engineered to localize to the cytoplasm for RNA cleavage. The CasRx expression cassette for cellular mRNA knockdown is shown in Figure 2A, and we next removed the two nuclear localization signals (NLSs) from CasRx (CasRx-del-NSL). By immunofluorescence detection, we found that CasRx-del-NSL was successfully expressed and mainly located in the cytoplasm (Figure 2C). As a control, CasRx was retained in the nucleus, as expected (Figure 2B).

To test the antiviral activity of the CRISPR-Cas13d system, we cotransfected CasRx-del-NSL with plasmid containing targeted crRNA or control crRNA in HEK293T cells. Twelve hours later, the transfected cells were infected with SVV at an MOI of 0.01 . As expected, we found that in the targeted crRNA group, there was extremely low expression of VP2 (which reflects the replication of SVV) in CasRx-del-NSL-expressing cells 

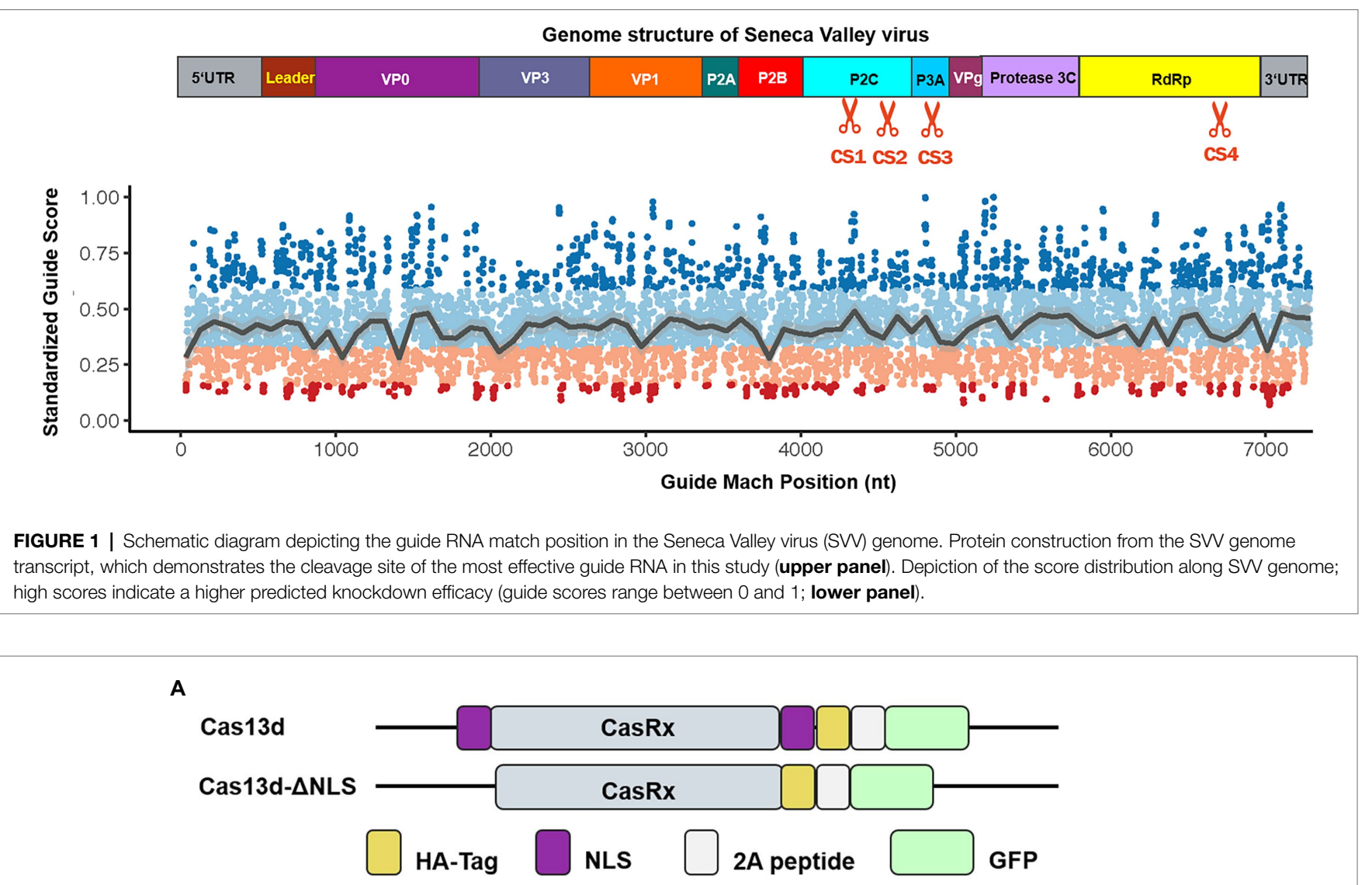

B
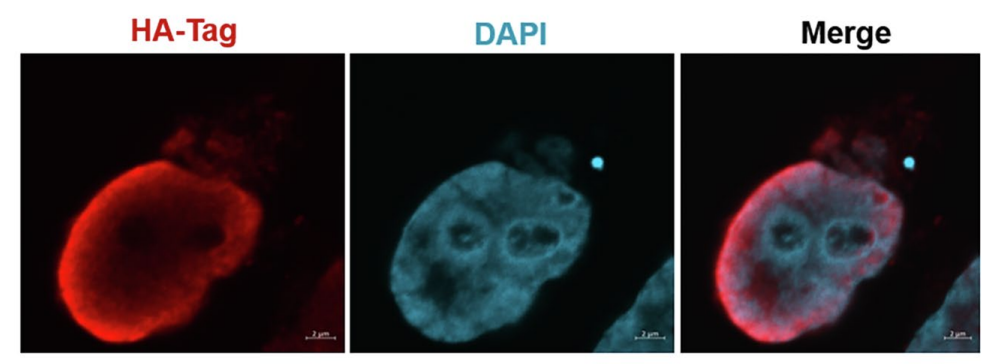

CasRx

C
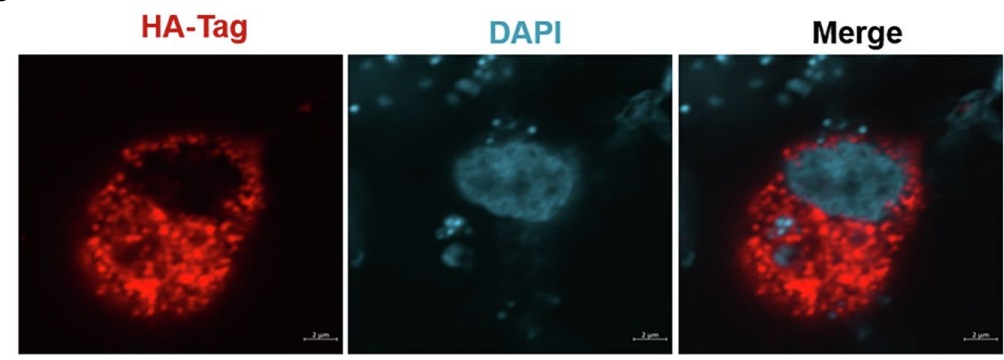

CasRx- $\triangle$ NLS

FIGURE 2 | Deletion of the nuclear localization signal (NLS) regulates Cas13d subcellular localization. (A) Summary of the constructs used in this study. Cas13d contains two NLSs that allow Cas13d to enter the nucleus. Removal of the NLS enables Cas13d to cleave the complementary target RNA within the cytoplasm but not the nucleus. (B) HEK293T cells were transfected with the CasRx expression vector or (C) CasRx-del-NLS, and then, IFA was performed at $24 \mathrm{hpi}$; the HA tag at the C-terminus of Cas $13 \mathrm{~d}$ was stained and labeled with Alexa Fluor 568-conjugated goat antibodies. Nuclei were labeled with DAPI. Scale bar $=2 \mu \mathrm{m}$. 
(Figure 3B); however, in the control crRNA group, VP2 and CasRx-del-NSL could be easily detected in the same cells (Figure 3A). The colocalization analysis was shown in Figure 3C. This indicated that the CRISPR/Cas13d system could efficiently inhibit SVV replication. To test the anti-SVV activity of four indicated crRNAs, we first evaluated the cell viability of indicated crRNAs transfected HEK293T cells, the result indicated that these crRNAs had not influence on cell viability at 24 and $48 \mathrm{~h}$ post-infection (Figure $4 \mathrm{~A}$ ). Next, we quantified the viral titers of the four indicated crRNAs and found that all of the

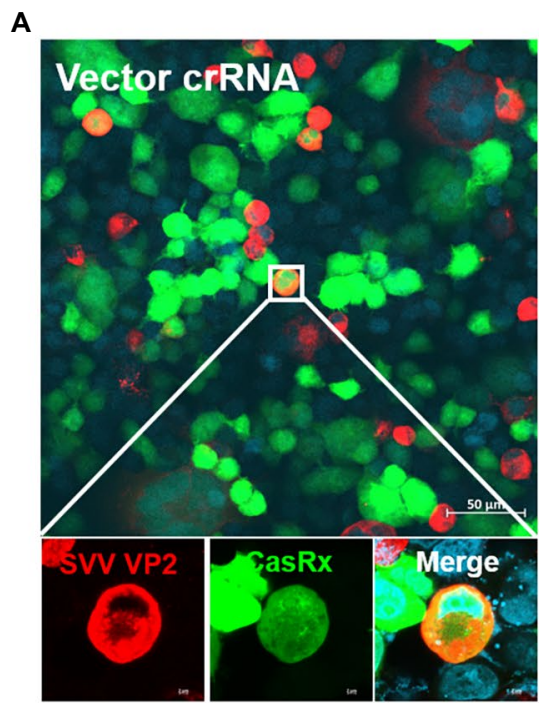

B

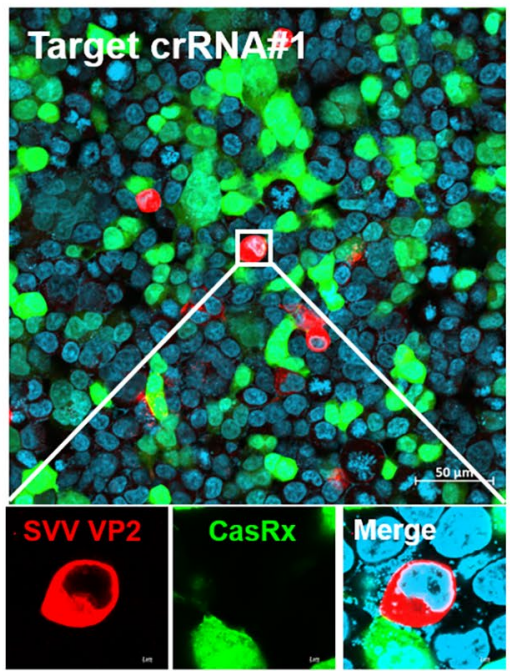

C

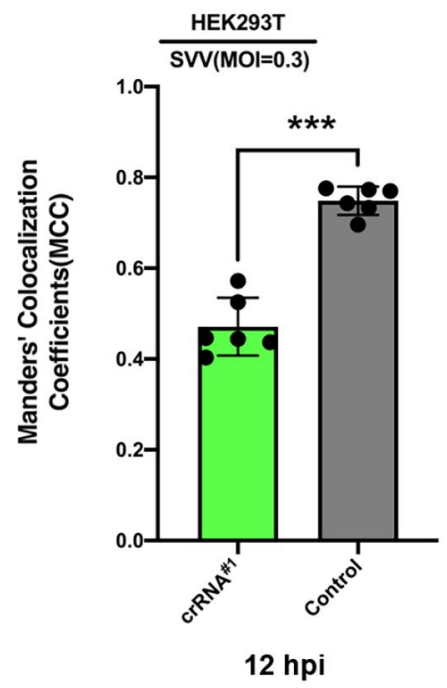

FIGURE 3 | Programmable targeting of SW RNA by Cas13d. The CasRx-del-NLS plasmid cotransfected with (A) control crRNA plasmid or (B) targeted crRNA\#1 plasmid into HEK293T cells. At $24 \mathrm{hpi}$, the cells were infected with SW (at an MOI of .3). Thirty-six hours post-infection, SW VP2 was detected by IFA with an antiVP2 monoclonal antibody and Alexa Fluor 568-conjugated goat antibodies. CasRx expression was indicated by EGFP, and nuclei were labeled with DAPI. (C) Manders' colocalization coefficients was analyzed, six random selected images were analyzed by ImageJ. ${ }^{\star \star \star} p<0.001$.

A
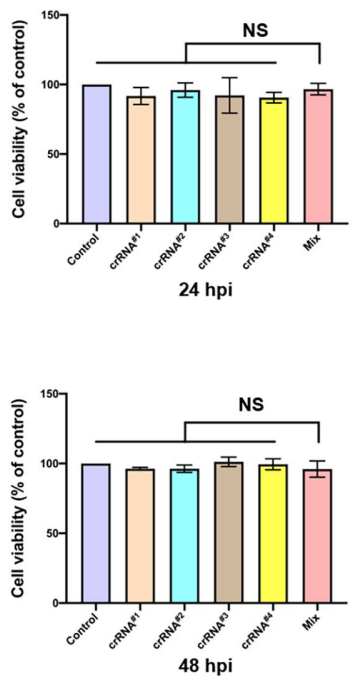

B

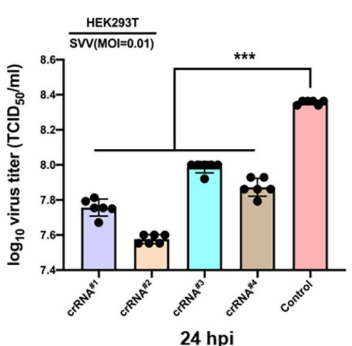

E

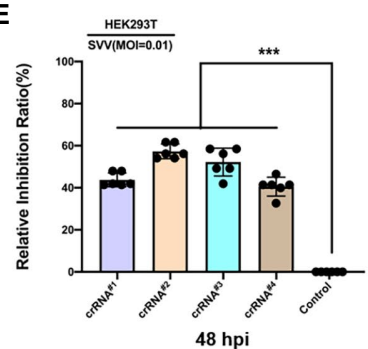

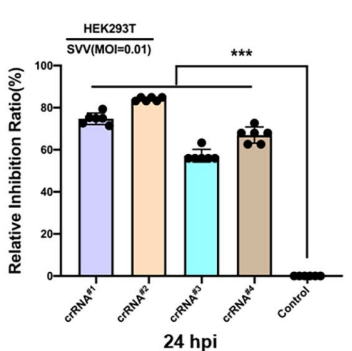

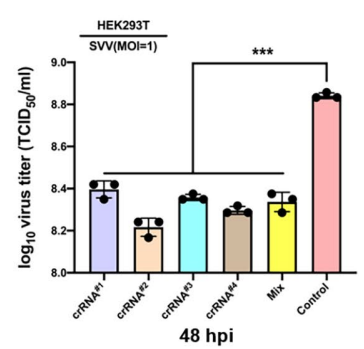

D

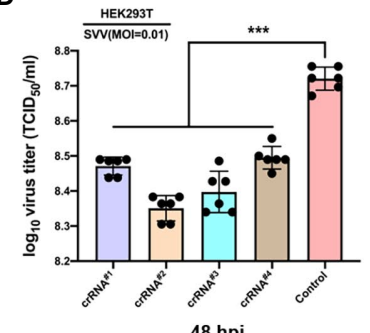

G

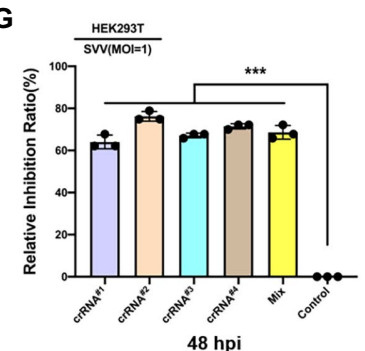

FIGURE 4 | Efficient inhibition of SW infection via the engineered CRISPR-Cas13d system. (A) HEK-293T cells were cotransfected with Cas13d and the indicated crRNAs plasmid, the cells viability at $24 \mathrm{hpt}$ or $48 \mathrm{hpt}$ was determined. (B) HEK-293T cells were cotransfected with Cas $13 \mathrm{~d}$ and the indicated crRNAs plasmid. Twenty-four hours later, the cells were infected with SWV (at an MOI of 0.01), and viral titers in the cell culture supernatants at $24 \mathrm{hpi}$ or (D) at $48 \mathrm{hpi}$ were determined. (C) The percentage reduction of virus titers was detected in the crRNA groups (\#1, \#2, \#3, and \#4) relative to the crRNA control group at 24 hpi or (E) at 48 hpi. (F) HEK-293T cells were cotransfected with Cas13d and the indicated crRNAs plasmid or crRNAs pool. Twenty-four hours later, the cells were infected with SW (at an $\mathrm{MOI}$ of 1 ), and viral titers in the cell culture supernatants at $48 \mathrm{hpi}$ were determined. (G) The percentage reduction of virus titers was detected in the crRNA groups (\#1, \#2, \#3, \#4, and mixed pool) relative to the crRNA control group at 48 hpi. Error bars represent the SD; ${ }^{\star} p<0.05$, ${ }^{\star \star *} p<0.001$. 
screened crRNAs significantly inhibited SVV replication at 24 and $48 \mathrm{~h}$ post-infection (Figures $4 \mathbf{B}, \mathbf{D}$ ). crRNA\#2 was the most effective crRNA among the four selected crRNAs. The viral titers of the crRNA\#2 group were significantly decreased (by 84\%) compared with those of the crRNA control group (Figures 4B,C). In addition, the titers decreased by $74 \%$ and $66 \%$ in the crRNA\# 1 and crRNA\#4 groups, respectively, compared with those in the crRNA control group (Figure 4C). All targeted crRNA groups showed a relative inhibition ratio of at least $57 \%$. At $48 \mathrm{hpi}$, the crRNA\#2 group maintained a robust ability to inhibit SVV replication, with a decrease of approximately $57 \%$ compared to the control group (Figures 4D,E). Furthermore, when compared with control crRNA, crRNA\#3, crRNA\#1, and crRNA\#4 reduced viral titers (by approximately 52\%, 43\%, and $40 \%$, respectively; Figures 4D,E). Next, we further evaluated the anti-SVV activity at a high MOI $(\mathrm{MOI}=1)$, we get a similar results as $\mathrm{MOI}=0.01$ (Figures $\mathbf{4 F , G ) . ~ W e ~ a l s o ~ e v a l u a t e d ~ w h e t h e r ~}$ these crRNAs had synergetic effect, to our surprised, we failed to observed synergetic effect as CRISPR-Cas9 system (Figures 4F,G; Tang et al., 2017). This needed to be explored in future. Overall, our results indicated that the crRNA consistently mediated robust Cas13d activity in infected cells. In fact, we also evaluate anti-SVV activity of CRISPR-Cas13d in porcine cells, however, all our tested porcine cells manifested a low transfection efficiency $(<30 \%)$. Therefore, we failed to perform this experiment in porcine cells, we speculated that lentiviral vectors will be an efficient transduction tool to test the anti-SVV activity of CRISPR-Cas13d in future. Furthermore, there are several issues should be concerned in future application, such as whether

\section{REFERENCES}

Abbott, T. R., Dhamdhere, G., Liu, Y., Lin, X., Goudy, L., Zeng, L., et al. (2020). Development of CRISPR as an antiviral strategy to combat SARS-CoV-2 and influenza. Cell 181, 865.e812-876.e812. doi: 10.1016/j.cell.2020.04.020

Burke, M. J. (2016). Oncolytic Seneca Valley virus: past perspectives and future directions. Oncolytic Virother. 5, 81-89. doi: 10.2147/OV.S96915

Flather, D., and Semler, B. L. (2015). Picornaviruses and nuclear functions: targeting a cellular compartment distinct from the replication site of a positivestrand RNA virus. Front. Microbiol. 6:594. doi: 10.3389/fmicb.2015.00594

Hales, L. M., Knowles, N. J., Reddy, P. S., Xu, L., Hay, C., and Hallenbeck, P. L. (2008). Complete genome sequence analysis of Seneca Valley virus-001, a novel oncolytic picornavirus. J. Gen. Virol. 89, 1265-1275. doi: 10.1099/ vir.0.83570-0

Hause, B. M., Myers, O., Duff, J., and Hesse, R. A. (2016). Senecavirus A in pigs, United States, 2015. Emerg. Infect. Dis. 22, 1323-1325. doi: 10.3201/ eid2207.151591

Horvath, P., and Barrangou, R. (2010). CRISPR/Cas, the immune system of bacteria and archaea. Science 327, 167-170. doi: 10.1126/science.1179555

Konermann, S., Lotfy, P., Brideau, N. J., Oki, J., Shokhirev, M. N., and Hsu, P. D. (2018). Transcriptome engineering with RNA-targeting type VI-D CRISPR effectors. Cell 173, 665.e614-676.e614. doi: 10.1016/j.cell.2018.02.033

Leme, R. A., Alfieri, A. F., and Alfieri, A. A. (2017). Update on Senecavirus infection in pigs. Viruses 9:170. doi: 10.3390/v9070170

Leme, R. A., Oliveira, T. E., Alcantara, B. K., Headley, S. A., Alfieri, A. F., Yang, M., et al. (2016). Clinical manifestations of Senecavirus A infection in neonatal pigs, Brazil, 2015. Emerg. Infect. Dis. 22, 1238-1241. doi: 10.3201/ eid2207.151583

Liao, F., Tang, Y. D., Tan, L., Yao, J., Zheng, C., and Wang, A. (2021). Using the CRISPR-Cas system to solve porcine viral infection-related issues. CRISPR J. 4, 776-788. doi: 10.1089/crispr.2021.0091 this CRISPR system has possible off-target effect and how to deliver it efficiently in vivo.

In summary, we demonstrated that the CRISPR-Cas $13 \mathrm{~d}$ system was a powerful tool to inhibit SVV replication. In the future, viral vectors or other potential CRISPR-Cas13d carriers may be used for SVV prevention in the clinic.

\section{DATA AVAILABILITY STATEMENT}

The raw data supporting the conclusions of this article will be made available by the authors, without undue reservation.

\section{AUTHOR CONTRIBUTIONS}

Y-DT, FM, and X-HC designed the experiments. Y-YZ and the other authors performed the experiments. Y-YZ, M-XS, YL, T-YW, M-YJ, CL, MC, Y-ZB, FM, X-HC, and Y-DT analyzed the data. Y-DT and Y-YZ wrote the manuscript. All authors contributed to the article and approved the submitted version.

\section{FUNDING}

This work was supported by grant from the National Natural Science Foundation of China to FM (32002249) and the State Key Laboratory of Veterinary Biotechnology of CAAS (SKLVBP201803 to Y-DT).

Lin, X., Liu, Y., Chemparathy, A., Pande, T., La Russa, M., and Qi, L. S (2021). A comprehensive analysis and resource to use CRISPR-Cas13 for broad-spectrum targeting of RNA viruses. Cell Rep. Med. 2:100245. doi: 10.1016/j.xcrm.2021.100245

Nguyen, T. M., Zhang, Y., and Pandolfi, P. P. (2020). Virus against virus: a potential treatment for 2019-nCov (SARS-CoV-2) and other RNA viruses. Cell Res. 30, 189-190. doi: 10.1038/s41422-020-0290-0

Reddy, P. S., Burroughs, K. D., Hales, L. M., Ganesh, S., Jones, B. H., Idamakanti, N., et al. (2007). Seneca Valley virus, a systemically deliverable oncolytic picornavirus, and the treatment of neuroendocrine cancers. J. Natl. Cancer Inst. 99, 1623-1633. doi: 10.1093/jnci/djm198

Reed, L. J., and Muench, H. (1938). A simple method of estimating fifty per cent endpoints. Am. J. Epidemiol. 27, 493-497. doi: 10.1093/oxfordjournals. aje.a118408

Tang, Y. D., Guo, J. C., Wang, T. Y., Zhao, K., Liu, J. T., Gao, J. C., et al. (2018). CRISPR/Cas9-mediated 2-sgRNA cleavage facilitates pseudorabies virus editing. FASEB J. 32, 4293-4301. doi: 10.1096/fj.201701129R

Tang, Y. D., Liu, J. T., Wang, T. Y., An, T. Q., Sun, M. X., Wang, S. J., et al. (2016). Live attenuated pseudorabies virus developed using the CRISPR/ Cas9 system. Virus Res. 225, 33-39. doi: 10.1016/j.virusres.2016.09.004

Tang, Y. D., Liu, J. T., Wang, T. Y., Sun, M. X., Tian, Z. J., and Cai, X. H. (2017). CRISPR/Cas9-mediated multiple single guide RNAs potently abrogate pseudorabies virus replication. Arch. Virol. 162, 3881-3886. doi: 10.1007/ s00705-017-3553-4

Wessels, H. H., Mendez-Mancilla, A., Guo, X., Legut, M., Daniloski, Z., and Sanjana, N. E. (2020). Massively parallel Cas13 screens reveal principles for guide RNA design. Nat. Biotechnol. 38, 722-727. doi: 10.1038/ s41587-020-0456-9

Yang, Y. L., Liu, J., Wang, T. Y., Chen, M., Wang, G., Yang, Y. B., et al. (2021). Aminopeptidase $\mathrm{N}$ is an entry co-factor triggering porcine deltacoronavirus entry via an endocytotic pathway. J. Virol. 95:e0094421. doi: 10.1128/JVI.00944-21 
Yang, Y. L., Meng, F., Qin, P., Herrler, G., Huang, Y. W., and Tang, Y. D. (2020). Trypsin promotes porcine deltacoronavirus mediating cell-to-cell fusion in a cell type-dependent manner. Emerg. Microbes Infect. 9, 457-468. doi: 10.1080/22221751.2020.1730245

Zhang, H. L., Li, Y. M., Sun, J., Zhang, Y. Y., Wang, T. Y., Sun, M. X., et al. (2021). Evaluating angiotensin-converting enzyme 2-mediated SARSCoV-2 entry across species. J. Biol. Chem. 296:100435. doi: 10.1016/j. jbc.2021.100435

Zhang, X., Zhu, Z., Yang, F., Cao, W., Tian, H., Zhang, K., et al. (2018). Review of Seneca Valley virus: a call for increased surveillance and research. Front. Microbiol. 9:940. doi: 10.3389/fmicb.2018.00940

Zhao, X., Wu, Q., Bai, Y., Chen, G., Zhou, L., Wu, Z., et al. (2017). Phylogenetic and genome analysis of seven Senecavirus A isolates in China. Transbound. Emerg. Dis. 64, 2075-2082. doi: 10.1111/tbed.12619

Zhu, Z., Yang, F., Chen, P., Liu, H., Cao, W., Zhang, K., et al. (2017). Emergence of novel Seneca Valley virus strains in China, 2017. Transbound. Emerg. Dis. 64, 1024-1029. doi: 10.1111/tbed.12662
Conflict of Interest: The authors declare that the research was conducted in the absence of any commercial or financial relationships that could be construed as a potential conflict of interest.

Publisher's Note: All claims expressed in this article are solely those of the authors and do not necessarily represent those of their affiliated organizations, or those of the publisher, the editors and the reviewers. Any product that may be evaluated in this article, or claim that may be made by its manufacturer, is not guaranteed or endorsed by the publisher.

Copyright (C) 2022 Zhang, Sun, Lian, Wang, Jia, Leng, Chen, Bai, Meng, Cai and Tang. This is an open-access article distributed under the terms of the Creative Commons Attribution License (CC BY). The use, distribution or reproduction in other forums is permitted, provided the original author(s) and the copyright owner(s) are credited and that the original publication in this journal is cited, in accordance with accepted academic practice. No use, distribution or reproduction is permitted which does not comply with these terms. 